\title{
Die rigorose Abfïhrbehandlung der Dysenterie, insbesondere durch medikamentös erzeugten osmotischen Transsudationsstrom in den Darm.
}

Von Prof. Theodor Hausmann in Moskau.

Zu der Mitteilung meiner'Erfahrungen in der Dysenteriebehandlung werde ich durch den Umstand veranlaßt, daß in dieser Frage tot nomia tot votá herrschen. Was die Serumtherapie anlangt, so ist in einem Punkt wenigstens eine Einigung erzielt, und zwar darin, daß die Dysenterie ein klinisch, nicht aber ätiologisch umschriebenes Krankheitsbild ist; sie kann durch die verschie- densten bakteriellen Erreger verursacht werden. Daher kann nur ein spezifisches, auf den im speziellen Fall aufgedeckten Erreger ein. gestelltes oder ein polyvalentes Serufn wirksam sein. In vielen Fällen ist aber der Erreger nicht zu eruieren, sodaB eine Serum. behandlung illusorisch wird. Einig ist man auch in dem Punkt, $\mathrm{da} B$ trotz der Spezifizität des Serums letzteres in einer nicht ge. ringen Zahıl von Fällen versagt, ebenso auch das polyvalente Serum. Hatte doch schon Rosenthal (D. m. W. 1904) 5\% Sterblichkeit bei Anwendung seines spezifischen Serums bei Kruse-Dysenterie. Ros en. thal war mit diesem Erfolg wohl nur deshalb zufrieden, weil in den anderen Moskauer Krankenhäusern die Mortalität etwa 10\% be. trug. Seine bessere Mortalitätsstatistik beweist nun aber nicht Wirk. samkeit des Serums, da sie die Folge einer besseren pharmakotherapeutischen Belrandlung gewesen sein kann. Die Verschiedenheit der Resultate der Autoren, die ihre Beobachtungen in den Kriegsjahren in der deutschen Literatur niedergelegt haben, beruht ja vielleicht auf einer Verschiedenheit der Sera, vielleicht aber mehr auf einer Verschiedenheit der daneben angewandten therapeutischen Maßregeln. Wemn wir Friede mann (D. m. W. 1917) Gehör schenken, so sind ceteris paribus die Heilerfolge oder Mißerfolge die gleichen, ob wir Serum anwenden oder nicht. Persönlich habe ich seit 1904 kein Serum mehr angewandt, aus dem einfachen Grunde, weil die von mir seit dieser Zeit geübte Behandlungsweise der Dysenterie eine Serumtherapie überflüssig macht.

Auf dem Gebiet der Pharmakotherapie der Bazillenruhr herrscht, wie auch das Studium der seit 1914 erschienenen deutschen Literatur und der Meinungsaustausch mit russischen Kollegen zeigt, eine Vielgestaltigkeit der Meinungen, wie sie sonst kaum auf einem anderen Gebiet der Therapie zu beobachten ist. Es scheint, daß die durch die Kriegsepidemie gezeitigten Massenbeobachtungen auch dem sonst klinisch geschulten Arzt nicht die Möglichkeit gaben, klinisch-therapeutische Fragen sicher zu entscheiden; in dieser Beziehung bieten aufmerksam und detailliert verfolgte Einzelfälle mehr Anbaltspunkte zur Entscheidung mancher therapeutischer Probleme. Im zivilisierten Deutschland hatten Kliniker und Aerzte nur ausnahmsweise Gelegen- 
heit, in Friedenszeiten Erfahrungen über Dysenteriebehandlung zu sammeln.

Meme Dysenterieerfahrungen erstrecken sich nun auf einen Zeitraum von menr als 25 Jahren. Ich verfüge sowohl über Massenbeobachtungen als auch über eine grolje $L$ ahl von gut verfolgten Einzelfällen. Die an Einzelfällen gewonnenen Eindrucke und Kesultate konnten am Massenmaterial geprüft werden, und umgekehrt. Wenn ich nun den Entwicklungsgaing, die Evolution meiner therapeutischen Grundsätze bei Dysenterıe verfolge, so finde ich für jede Etappe dieser Evolution eine entsprechende Vertretung in der seit 1914 erschienenen deutschen Literatur, sowohl für die Antangsstufe als auch tür die Mittelstufen meiner Evolution. Auch die Endstufe dieser Evolution finde ich, wenigstens im Prinzipe, in den Arbeiten einiger weniger Autoren.

Der Beghin meiner ärztiicnen Tätigkeit ist durch eine stopfende Behandlung der Dysenterie mit $O$ pi um und $W$ is mut und mit $T$ anninklistle re n markiert. Der Erfolg befriedigte mich nicht. Todesfälle waren keine Seltenheit. Der Ersatz des Wismuts durch Tannalbi n änderte nichts an der Sa'che. Mehr Befriedigung erhielt ich, als ich die Tanninklistiere durch Amylumklistie re und besonders Le insamenklistiere zu ersetzen begann; doch war der Erfolg mehr symptomatisch, ein wesentricher Einflus auf den Verlauf und den Ausgang der Erkrankung konnte von mir nicht mit Sicherheit konstatiert werden. Die Kombination der Tinctura opii mit Tinctura bell ad on nae brachte dann den Kranken bedeutend mehr Erleichterung als Opium allein. Doch alles das schien mir bloß palliativ zu wirken.

Die zweite Hauptetappe in meiner Dysenterieerfahrung ist durch die Verordnung eines Abführmittels zum Beginn der Be. h andlung gekennzeichnet, worauf erst die von mir bisher geübte Behandlungsweise einsetzte. In der ersten Zeit gab ich Rizinus am ersten Tag der Behandlung. $\mathrm{Da}$ danach die Besserung meist nur kurzdauernd war, ging icn dazu über, bei Dysenteriekranken am ersten Behandlungstage $\mathrm{Kal}$ om el zu verordnen. Ich gab morgens früh 2 Pulver Kalomel à 0,2 in einem $Z$ wischenraum von zwei Stunden und 2 Stunden nach dem 2. Pulver Rizinus. Der Erfolg war ausgezeichnet, besonders als ich gleichzeitig eine absolute Nahrungsabstinenz einführte und den Kranken während einiger Tage nur Tee mit Zucker zu trinken gab. Wurde diese Behandlung in den ersten Tagen der Erkrankung eingeleitet, so wurde sie meist prompt kupiert, sodals ich anfing, auf Klistiere ganz zu verzichten. Während ich anfangs glaubte, nach dem Kalomeltage wieder Stopfmittel geben zu müssen, habe ich später auf diese vollständig verzichtet. Wetn aber nach einem Kalomeltage die Dysenterie noch nicht behoben war, so wiederholte ich 1- bis $2 \mathrm{mal}$ die Kalomel-Rizinusbehandlung mit $1-2$ freien Zwischen-tagen. Die Kalomelkur galt als gelungen, wenn durch sie eine ausgiebige Abführwirkung erzielt wurde mit Abgang nicht nur von Schleimmassen und Blut, sondern auch von reichlichen Kotmassen. Gelegentlich $\mathrm{kam}$ es mir aber vor, daß solch eine ausgiebige Entleerung nicht zu erzielen war, trotz Wiederholung der Kalomel-Rizinusgaben. In solchen Fällen zog sich die Krankheit sichtlich in die Länge. Dieser Unrstand veranlaßte mich, das Rizinus, unter Beibehaltung des Kalomels, durch GIa ube rs a I z zu ersetzen. Ich gab 4 Stunden nach der 2. Kalomelgabe $15,0 \mathrm{Natrium}$ silfuricunt siccum in 1 Glas Wasser. Schion der erste Versuch gab ein geradezu verblüffendes Resultat. Die kolossale wäßrige Entleerung schaffte verflüssigten Kot und viel Schleim- und Gewebsfetzen heraus. Die Entleerung war so ausgiebig und voll, wie ich sie vorher nie gesehen hatte. Die Dysenterie wurde danach geradezu kupiert

Seitdem wende ich bei jeden 1 Dysenteriefall Glaubersalz in gehäuften Mengen an. In letzter Zeit habe ich gelegentlich Glaubersalz allein ohne vorhergehende Kalomelgaben verordnet mit gutem $\mathrm{Er}$ folg. In frischen Fällen genügt meist eine einnalige Kalomel-Glatbersalzverordnung und schafft ohne jede andere Verordnung Heilung. In älteren Fällen muß das Glaubersalz in gleichen Gaben 1- bis 3mal wiederholt werden unter $Z$ wischenschaltung von freien Tagen.

Auch bei dieser Medikation halte ich das Einhillten von absoluter $\mathrm{Nahrungsabstinenz}$ wälirend einiger Tage unter Verabfolgung von Tee mit Zucker und vielleicht Bouillon für durchaus notwendig. Um so schneller köınen wir dann zu einer kräftigenden Ernährung übergehen. Auffallend ist, daß die Tenesmen schon vor der Ausleerung nach Glaubersalz nachlassen und nach der Entle rung fortbleiben. Selbst dann, wenn in veralteten Fällen, nach einer Glaubersalzentleerung dysenterische Schleimstühie noch fortbestehen, werden die Kranken meist nicht mehr von Tenesmen geplagt, wodu'rch sich die Glaubersalzabführung vorteilhaft vor der Kalomelund Rizinusabführung unterscheidet: Wenn nach letzteren Mitteln die dysenterischien Schleimstüllle fortbestehen, so bestehen auch die Tellesmen weiter.

Die Glaubersalzbehandlung wird auch sonst von einigen Autoren empfohlen, jedoch unter Einhaltung einer anderen Technik; es wird das Salz zu 3-4 g genau mehrere Male täglich durch einige Tage hindurch verördnet. Bei dieser Verordnungsweise ist die Wirkung eine ganz andere. Zwar erreichen wir dabei meist eine mehr oder weniger gute Abfiihrwirkung, doch nie erzielen wir eline so vollkommene Entleerung und Reinigung des Dickdarmes, wie bei einmaliger großer Dosis. Aber auch wenn das Salz refracta dosi gegeben wird, zeigt sich eine günstige Einwirking auf die Tenesmen und den Stuhlgang. Diese werden seltener und schwächer. Bei
dieser Gelegenheit möchte ich auf eine niclit selten von mir beob- achtete Erscheinung bei enteritischen (nicht dysenterischen) Durchfällen aufmerksam machen, wenı Giaubersalz in kleineren Dosen gegeben wird: die Zahl der Entleerungen wird dabei nicht häutiger; sondetn seltener.

Wie dem auch sei, ich nehme mir das Recht, zu behaupten, daß die einzige rationelle Behandlung der Dysenterie in einer rigorosen, furchtlosen Abfuhrung besteht. Ich stütze mich auf eine großße Zahl von Einzelbeobachtungen, die mir diese Wirkung unmittelbar vor Augen fülnten. Auch meine Mortalitätsstatistik gibt mir das Recht zu einer solchen These.

Bis zum Jahre 1904, wo ich mit Scopimittelu, Adstringentien und Klismen die Dysenterie bekämpfte, eventuell unter Verordnung einer einmaligen Rizinusgabe zu Beginn der Behandlung, habe icn eine ganz erkleckliche Lahl von Todesfällen, erlebt. Auch bei günstigem Ausgang währte das Leiden oft mehrere Wochen, um nicht selten in ein chronisches Stadium überzugenen. Se it 190t, wo ich zur Kalomel-Rizinus-bzw. Glaubersalztherapie unter Nahrungsabstinenz überging, habe ich unter vielen hunderten Fällen von typischer Dysenterie, die zu Beginn des Leidens in meine Hände kamen, nicht einen einzigen Todesfall gehabt. - Die 3 Todesfälle, die ich seit 1904 erlebt habe, beziehen sich auf Fälle, die ich nach längerer Krankheitsdauer in desperatem $\mathrm{Zu}$ stande in die Hände bekam. Alle drei waren mit Stopfmitteln, Adstringentien und Klistieren vorbehandelt worden. Es gelang nicht, diese Kranken zu retten, trotz zeitweiliger Besserung, die durch die Abführung erzielt wurde. Die Todesfälle sind nicht auts Konto der Abführbehandlung zu setzen, sondern sie sind der Vorbehandlung zuzuschreiben, welehe durch Stopfmittel den toxischen und gewebsschädigenden Darminhalt durch Wochen hindurch dem Kranken erhalten hat, anstatt ihn hinauszuschaffen.

Wenn wir von dem katarrhalisch-enteritischen Stadium absehen, welches durch echte Diarrhöen charakterisiert ist, ist die typische, vollentwickelte Dysenterie funktionell als Obstipation aufzufassen, wie aus Folgendem klar hervorgeht.

1. Es enthalten die schleimigen Entleerungen keine Kotteile, daher sind sie geruchlos.

2. Bei der Palpation des Dickdarms, die nach den Prinzipien der von mir geübten und in meinem Buche "Die methodische $\mathrm{G}$ astrointestinalpalpation, 2. Aufl. 1918, S. Karger" beschicebenen Methode der topographischen Gleit- und Tiefenpalpation ausgeführt werden muß, vermissen wir die Zeichen einer Diarrhoe, d. h. die Palpation deckt keinen flüssigen Dickdarm inhalt auf. Es läBt sich weder Plätschern, noch Quatschern, noch großblasiges Gurren erzeugen. Die einzelnen Abschnitte des Dickdarms, der Flexura sigmoidea, des Colon transversum, erscheinen gut kontrahiert und lassen teilweise, so besonders an der Flexura sigmoidea, an der derben, wurstförmigen Gestalt erkennen, da 3 sie feste Kotmassen beherbergen. Das Zökum ist im Anfangsstadium der Dysenterie noch von flüssigem Inhalt erfüllt und weist dann akustische Phänomene bei der Palpation auf; später aber läßt auch das Zökum akustische Phänomene vermissen und erscheint stark kontrahiert. Gelegentlich ist die Flexur frei von Kotmassen, leer, als kleinfingerdicker Strang palpierbar, oft auch das Transversum.

3. Bei der Röntgenuntersuchung sieht man, daß der Wismutbrei den Dünndarm äußerst langsam passiert. Besonders lange hält sich der Kontrastschatten vor dem Eintritt ins Zökum auf, ein Zeichen, daß der lleozökalsphinkter spastisch kontrahiert sein muB. Wird dann das, Zökum langsam gefüllt, so erscheint es von geringem Durchmesser, also ebenfalls tonisch kontrahiert. 24 Stunden nach Einnahme des Wismutbreies hat dieser den proximalen Abschnitt des Dickdarms noch nicht verlassen. Weiter die Beobachtung am Röntgeuschirm zu führen, habe ich für unangebracht gehalten, aus Gründen der therapeutisch notwendigen Maßnahmen, d. h. Abführung, um so mehr, als die gewommenen Resultate hinlänglich beweisen, daß bei der Dysenterie die Passage durch den Darm erheblich verlangsamt ist.

Daraus ergibt sich, daB die Verordnung von Opium und anderen Stopfmitteln bei Dysenterie direkt kontra. indiziert ist, um so mehr, als Opinm, Morphium und Kodei (Pal, Poppert u. a.) die zirkuläre Darmmuskulatur zur Kontraktion bringen und den bei Dysenterie ohnehin vorhandene Darmspasmus nur verstärken. Da Opium den Defäkationsreflex hemmt (d e Querva in, Magnus), so kann das Opium nur symptomatisch wirken, indem es zeitweilig die schmerzhaften Tenesmen mildert.

Die Dysenterie muß also mit Abführmiteln behan. delt werden ${ }^{1}$ ). Nun hat mir aber die Erfahrung gezeigt, daß es durchaus nicht gleichgültig ist, welch ein Abführmittel wir wählen Die experimentellé Pharmakologie zeigt uns denn auch daß die pharmakodynamische Wirkung der einzelnen Abführmittel durchaus verschieden ist.

Das Ol. ricini, oder vielmehr die aus ihr durch Lipasewirkung abgespaltene Rizinolsäure, erregt die Dünndarmperistaltik. Der flüssige Dünndarminhalt entgeht der Resorption und über

1) Autoren alter Schule, so Heubner in Zl em Bens Handbuch, heben mit Nachdruck die Abfuhrbehandlung der Dysenterie hervor. Auch $S$ tr ü mpell spricht schon in den achtziger Jahren in seinem Lehrbuch von der B hardlung der Dysenterie mit Rizinus wie von etwas Allgemeinbekanntem. Diese Methode scheint aber, der neueren und neusten Literatur nach zu urteilen, meistens verkannt oder vergessen worden zu sein und mubte sozusagen neu entdeckt. ihr. Nu'zen von' neuem hewiesen werden. sein und mubte sozusagen neu entdeckt. ihr Nu'zen von' neulem hewiesen werden.
Die guten alten Mittel werden hoffentllch uber de modernen. Pro lukte der Arzneiz mittelindustrie verdienterweise einen Sieg davontragen. 
schwemmt das Zobum und den Dickdarm, wobei die Antiperistaltik im proximalen Kolon aufgehoben wird. Der flüssige Dickdarminhalt gelangt nun schnell in den distalen Dickdarm und das Rektum, von wo aus der Defäkationsreflex ausgelöst wird ( $M$ a g nus).

Senna, Rheum, Cascara und andere Kathartinsäurederivate enthaltende Drogen lösen einen Defäkationsreflex vom Zökum aus unter Aufhebung der Antiperistaltik (Magnus, Stierlin). Dem Rizinusöl wie der Sennagruppe ist das gemeinsam, daB der eingedickte Kot nicht verflüssigt wird, sondern er wird zusammen mil dem flüssigen Kot ausgestobsen. Die Entleerung des Kolons ist nur unvollkommen.

Die Mittelsalze (Natrium sulfuricum, Magesium sulfuricum) regen nach den Untersuchungen von Best die Peristaltik nicht nur des Dünndarms, sondern auch des Dickdarms an. AuBer dem erfolgt eine Flüssigkeitsausscheidung in das Darmlumen, und zwar eine osmotische Transsudation einer eiweiBarmen Flüssigkeit (Ury). Da die Antiperistaltik nicht aufgehoben ist, wird das Sal längere Zeit im proximalen Kolon aufgehalten, wodurch die Transsudation längere Zeit unterhalten wird. Der Dickdarmirthalt wird vollständig verflüssigt, der Dickdarm, wie auf dem Röntgenschirm zu sehen ist, vollständig entleert ( $\mathrm{Pad}$ tberg).

Weñiger erforscht ist die Wirkung des Kalomels. Nach Babkin reizt das Kalomel den Dünndarm zu stärkerer Sekretion an. Nach Flexeder wird die Peristaltik angeregt und dabei die Resorption im Dünndarm gestört. Die Praxis lehrt, daß feste Kotmassen nicht verflüssigt werden. Beiläufig mache 'fch auf die kalkfällende und dadurch erregungssteigernde Wirkung des Kalomels aufmerksam. Von allen erwähnten Abführmitteln habeț Mittelsalze die vollständigste entleerende Wirkung. Diese ist mit einer Dauerspülung des Dickdarms zu vergleichen. Die Beobachtungen bei der Dysenteriebehandlung entsprechen vollkommen den Resultaten der experimentellen Pharmakologie. Bei der durch groBe Glaubersalzdosen hervorgebrachten Dauerspülung wird der erkrankt Dickdarnı von Schleim, Eiter, Blut, Bakterien und Kot gut gereinigt, wie eine eiternde Wunde der Extremitäten bei einem Dauerbad.

Daneben läßt sich eine sehr erwünschte, bisher noch nicht vermerkte Nebenwirkung erkennen: gleichzeitig mit der Verflüssigung des Darminhaltes, erkennbar an dem palpatorisch festzustellenden Plätschern und Quatschern im Dickdarm, kommen wir durch Palpation konstatieren, daß der vorher stark kontrahierte Dickdarm weit und schlaff wird, der Tonus und die spastische Kontraktion lassen sichtlich nach. Dadurch erklärt sich das $\mathrm{Nachlassen} \mathrm{der} \mathrm{Te-}$ nesmen schon vor der Entleerung und das Aufhören derselben nach der Entleerung. Die Kranken merken eine auffallende Erleichterung schon. bald nach der Einnahme des Glaubersalzes. Manche geben an, es wäre ilinen, als würde ein die Därme zuschnürender Strang gelöst werden. Das Nachlassen der Spasmen hat eine günstige Wirkung auf den Heilprozeß, denn der Darmkrampf, welcher durch den EntzündungsprozeB hervorgerufen wird, unterhält und steigert den entzündlichen Zustand der Darmwand.

Wenn wir einen frischen Fall vor uns haben, so genügt oft eine einzige große Glaubersalzgabe, um die Dysenterie zu kupieren, in anderen, besonders älteren Fällen muB diese Medikation 2-' bis $3 \mathrm{mal}$ wiederholt werden. So kommen wir ganz olne andere. Medikamente und ganz olıne Klistiere aus. In kurzer Zeit wird der Stuhl fest, in schweren Fällen, die schon eine längere Krankheitsdauer hinter sich haben, in etwa 1 Woche, sonst aber nicht selten schon in 3-4 Tagen. Kam der Kranke nach längerer Krankheitsdauer in Behandlung und war der Proze B ein schwerer, so können neben dem festen Stuhl noch Schleimklumpen entleert werden. Es ist in solchen Fällen angebracht, in längeren $Z$ wischenräumen durch große Glaubersalzdosen den Dickdarm auszuspülen. Daneben bewähren sich heiBe Klistiere von $37^{\circ}-38^{\circ} \mathrm{R}$. 1/4-1/2\%iger Kalihypermanganicumlösung.

Ein Chronischwerden der Dysenterie wird bei der beschriebenen Beliandlungsart kauri jemals beobachtet.

Da eine starke Transsudation nur dann stattfinden kann, wenn der Wassergehalt des Körpers und der. Gewebe genügend ist (Meyer-Gottlieb, Experimentelle Pharmakologie), und ganz ausbleiben kann, wenn dem Körper mehrere Tage keine Flüssigkeit zugeführt worden ist, so muß die Regel gelten, den Kranken von dem Moment an, wo man ilun in Behandlung bekommt, viel trinken $\mathrm{zu}$ lassen. Bei augenscheinlicher Wasserarmut des Körpers, wie sie nach längerem Krankenlager gelegentlich beobachtet wird, ist es ratsam, vor und einige Zeit nach der Glaubersalzgabe Infusionen von physiologischen Lösungen zu machen (Locke-lösung). Letzteres ist auch bei starker Herzlabilität angebracht, um es zu verhüten, daß die Wasserentziehung die GefäBfüllung so weit herabsetzt, daB das Pumpwerk des Herzens versagt.

Eine Kontrolle mittels des Rektoskops ist nicht vonnöten. Doch ist es interessant, den Heilungsvorgang rektoskopisch zu verfolgen. Während vor der ersten Glaubersalzgabe meistens das Ein. führenl des Rektoskopes wegen des Sphinkterspasmus nur ausnahmsweise gelingt, ist dieses nach der Glaubersalzwirkung leicht möglich. Das Rektoskop zeigt uns die vorsichgehende Reinigung der Dickdarmschleimhaut und bei wiederholter Rektoskopie den schnellen Fortschritt derselben. Keine einzige andere Behandlungsmethode zeitigt solche Erfolge. Die Mittelsalze wirken geradezu spezifisch nicht nur durch physikalische Reinigung des Dickdarms; der osmotische starke Strom ruft zweifel. los eine gewaltige heilbringende Umstimmung der Darmwand hervor. Der osmotische Transsudations. strom muB verständlicherweise die mechanische Los. lösung nekrotisierter oberflächlicher Schleimhat schichten, welche massenhaft Bakterien enthalten, bewirken. Mit dem Strom werden auch Toxine und schädliche Stoffwechselprodukte ausgeschieden. SchlieBlich ist der Umstand von Bedeutung, daB nach den Untersuchungen von Holzinger (Berl. klin. Wschr. 1909, 3 und Protokolle des I. Baltischen Aerztekongres. ses in Dorpat 1910) der osmotische Strom die Entwick. lung und Lebenstätigkeit der Bakterien hemmt, so. daB die in den tieferen Schichten der Schleimhaut und Submukosa befindlichen Bakterien (s. Zieglers Spez. Pathologische Anatomie) durch den Strom nicht nur ausgeschwemmt werden können, sondern auch bedeu. tend geschädigt werden. In einem von mir angestell. ten Versuch gelang es nicht, eine Katze mit der nach Glaubersalz erfolgten Entleerungeines Dysenterikers zu infizieren.

Hervorzuheben ist, daB nichtfiebernde Dysenteriker - zu denen nicht selten gerade die schwereren Fälle gehören - nach einer energischen Abführung mit kurzdauerndem $\mathrm{Fi}$ i be $\mathrm{r}$ reagieren. Das ist ein Zeichen, daB die Abfülrung in innere Lebensvorgänge eingreift.

Nach allem ist die Verordnung des einen oder des anderen Abführmittels nicht nur Geschmackssache; sie wird durch die pharmakodynamische Bedeutung der einzelnen Mittel vorgezeichnet. Die Verordnung des Glaubersalzes bei Dysenterie ist also nicht nur empirisch erprobt, sondern auch durch pharmakologische Tatsachen ohlbegründet.

Die Mittelsalzbehandlung der Dysenterie zeigt nun, daB bei rechtzeitiger Anwendung ein Chronischwerden der Dysenterie nicht vorkommt. Auch in anderer Beziehung macht sich ihr Einflul auf das Nachstadium bemerkbar. Bei der Dysenterie finden wir meist eine Anazidität und oft eine vollständige Achylie, welche noch lange Zeit nach Heilung der Dysenterie fortbesteht. Die Mittelsalze rufen nun eine starke Transsudation auch. in dem Magen hervor. Dadurch ist es zu erklären, daB nach Mittelsalzbehandlung die Sekretionsverhältnisse relativ schnell wieder zur Norm zurückkehren, denn der osmotische Strom ruft auch in der Magenschleimhaut eine Umstimmung hervor.

Somit ist die energische Abführbehandlung, speziell die mit Mittelsalzen, wie Glaubersalz, die Methode der Wahl bei Dysenterie. Auf sie zu verzichten, weil sie den Kranken schwächen könne oder eine schon bestehende Schwäche vergrößere (S a l o mon, W. kl. W. 1915), ist nicht richtig ill Anbetracht der großèn Vorteile, die diese Therapie mit sich bringt. Etwaigen. Schädigungen kann durch I n fusio nen vorgebeugt werden (s. oben), eventuell mit Adrenalinzusatz.

Wird einmal mit Glaubersalz keine ausgiebige Entleerung erzielt so kombinieren wir es mit protrahierten Rizinusgaben (2mal täglich 1 EBlöffel)

Von anderen sonst empfohlenen Mitteln - Wismut Tannabin, Bolus, Kohle usw. - hat keines mich befriedigt. Doch eine Ausnahme möchte ich machen, und zwar mit dem A tropin. Mit Umber Schwartze (beide Arbeiten s. D. m. W. 1917) halte ich das Atropin' für ein ganz vorzüglich auf die Schmerzen und Tenesmen einwirkendes Mittel, da es die Spasmen behebt.

Mit dem Morphium, Opium, Pantopon oder ähnlichen Präparaten (mit Ausnahme des Papaverins, welches ähnlich, abe schwächer wirkt als Atropin) erreichen wir diesen Zweck niclit Zwar wird die Schmerzempfindung gemildert, doch wegen der kontraktionsvermehrenden Wirkung der Opiumpräparate (Pal, Pop pert, Magnu s) werden die Spasmen nicht gelöst.

Das Atropin unterstützt in nicht zu verkennender Weise den HeilprozeB, sofern die Spasmen diesen hinderlich sind. Doch muß ich sagen, daB wir bei der Glaubersalzbehandlung sehr woh ohne Atropin auskommen können, da die osmotische Transsudation mit einem Tonusabfall der Darmwand einhergeht, welcher der Atropin wirkung ziemlich gleichwertig ist.

Tatsächlich habe ich das Atropin neben dem Glaubersalz nur selten anzuwenden brauchen. Seit vielen Jahren verordne ich abe statt Opium die Tinctura belladonnae zu $10-20$ gutt. mit zufriedenstellendem Erfolg

Von mancher Seite wird Adrenalin bei Dysenterie empfohlen. Selbst habe ich darüber keine Erfahrung. Doch kann ich es mi sehr wohl denken, daß das Mittel bei dem durch den Infektions prozeB erschöpften Adrenalsystem substituierend wirkt und besonders dort ángezeigt ist, wo der Zirkulationstonus gefallen ist Als splanchnikusreizendes Mittel muB es den krankhaft erregten Tonus des Darmes mindern und daher antispasmodisch wirken.

Was die Diät anbetrifft, so habe ich mich an das Prinzip gehalten, daB, je rigoroser innerhalb kurzer Zeit die Nahrungsentziehung ist unter Zuführung von Flüssigkeiten (Tee mit Zucker), desto schneller ein Zustand eintritt, der eine kalorienreichere Ernährung gestattet. Der Krankheitsverlauf wird dadurch wesentlich abgekürzt. Die Gesichtspunkte, die beim Typhus uns veranlassen, den Kranken von Beginn an gut $z u$ eruähren, sind bei der Dysenterie nicht maB gebend, denn beim Typhus hat das Hungern keinen EinfluB auf die 
Erkrankung des Darmes, wohl aber bei der Dysenterie, die vor allem eine ideale Entleerung und Reinigung des Darmkanals erfordert. Deshalb verzichte ich auf ein paar Hungertage auch in allen in der Ernährung bereits herabgekommenen Fällen nicht. Wenn dann später Inanitionsödeme eintreten, so'sage ich mir erstens, daB diese auch dann eingetreten wären, wenn ich die Hungertage nicht eingeschoben hätte, da dann die Krankheit eben länger gewährt hätte. Zweitens sage ich mir, daB ich die Inanitionsödeme bald durch geeignete kalorien- und vitaminreiche Ernäh. rung fortschaffen: kann, wenn erst die Verdauungsorgane eine reichliche Ernährung gestatten. Das Hauptziel ist das, da $B$ wir so oder anders den KrankheitsprozeB am Darm beheben. Was hilft auch eine allzufrüh begonnene kräftige Ernährung, wenn die Nährstoffe infolge der meist vorhaidenen vollkommenen Achylie und Apepsie der Verdauungssäfte ${ }^{1}$ ) nicht verdaut werden, sondern der Gärung und Fäulnis anheimfallen und dadurch bloB neue Schädigungen des Verdauungskanals veranlassen.

Aus diesem Grunde wird ja erfahrungsmäBig selbst die Milch von Dysenterikern so schlecht vertragen, denn das Kasein gerinnt nicht im Magen und verfällt abnormen Spaltungsvorgängen. Der Milchzucker, welcher überhaupt von Erwachsenen schwer invertiert wird, vergärt. Ganz schlecht werden auch Eier vertragen, indem das Eigelb viele Fäulnisprodukte 'liefert. Daß seit altersher Dysenterikern mit Vorliebe aufgeschlossene Kohlenhydrate gegeben werden, hat seinen triftigen Grund, denn sie unterliegen am wenigsten der Gärung. Doch das nur beiläufig, da es nicht meine Aufgabe war, das diätetische Regime bei Dysenterie darzustellen.

Jetzt noch einige Worte zur Frage der Klismenbehandlung der Dysenterie. Es gab eine Zeit, wo ich glaubte, das Heil läge in der topischen Beeinflussung des Dickdarms durch medikamentöse Klismen. Doch als ich mit Hilfe meiner Gleit- und Tiefenpalpation systematisch den Dickdarm auch bei Dysenterikern zu untersuchen begann, wurde es mir offenbar, daß in der Regel die Flüssigkeit nicht in den Dickdarm gelangt, wie es sonst der Fall ist. In der Flexur wie auch in den höheren Dickdarmabschnitten tritt nach dem Klisma bei Dysenterikern kein Quatschern oder Plätschern auf - ein Zeichen, aaß die Flüssigkeit vor dem kontrahierten Sphincter rectoromanus haltmacht und in der Ampulle verbleibt. Die Sachlage ändert sich aber, wenn wir vorher Atrop in einspritzen, oder wein wir eine Abftihrung durch Gla ubersalz bewirkt haben. In heiden Fällen ist der Sphinkter so weit erschlafft, daß die Klismenflüssigkeit nunmehr auch in den Dickdarm gelangen kann, was palpatorisch unschwer erkannt werden kann. Die gleiche Beobachtung köınen wir auch auf dem Röntgenschirm machen. Kontrastflüssigkeit gelangt gar nicht oder zu einem nur sehr geringen Teil in die Flexur. Nach Atropin, besonders aber nach Glaubersalzabführung füllt sie den Dickdarm. Ist der Sphinkterspasmus gelöst, so wende ich mit Vorteil heiße $\left(37-38^{\circ} \mathrm{R}\right)$ Amylumklismen mit Zusatz von 20 gutt. Jodtiriktur an. Tanninklistiere wende ich längst nicht mehr an.

Ergebnisse. 1. Die einzig rationelle Behandlunsmethode dier Dysenterie ist die rigoros und systematisch abführende.

2. Die vollständigste Entleerung des Dickdarms wird durch gehäufte Glaubersalzdosen $(15,0-20,0$ Natrii sulfurici sicci) erzielt, die $1-, 2-$ bis $3 \mathrm{mal}$ zu verabfolgen sind. Es wird eine gewaltige Transsudation in den Dickdarm und dadurch eine Dauerspülung desselben erzielt, wobei der Spasmus der Darmmuskulatur aufgehoben wird. Der ersten Glaubersalzgabe soll man eine kräftige Kalomelgabe voratusschicken.

3. Stopfmittel sind kontraindiziert, da es bei der typischen Dysenterie sich um eine Obstipation handelt. Besonders ist Opium zu vermeiden.

4. Adstringentien sind überflüssig und helfen nichts.

5. Adsorbentien (Bolus, Tierkohle) sind in ihrer Wirkung unsicher. Sie sind auch bei der energisclien Abführbehandlung mit Glaubersalz überflüssig.

6. Atropin ist ein vorzügliches symptomatisches Mittel bei Dysenterie.

7. Der Krankheitsverlauf wird durch Einschaltung einiger Hungertağe abgekürzt.

8. Medikamentöse Klismen sind im akuten Stadium zwecklos.

9. Wenn bei einer Behandlung der Dysenterie mit Stopfmitteln, Adstringentien und Klismen in vielen Fällen die Krankheit schlieBlich glücklich überstanden wird, so beweist das nur, daB die Fähígkeit des Körpers zur Selbsthilfe groß ist.

10. Von der Serumtherapie dürfen wir bei der Abführbehandlung absehen. Die Erfolge der ersteren sind unsicherer. als die der letzteren.

11. An Dysenterie darf ein Kranker, der nicht gerade zu spät in Behandlung kommt, nicht sterben.

1) Uffelman n hat schon 1874 (Arch. f. klin. M.) beobachtet, daß bei einer mit einer Gallenfistel behafteten Kranken während einer interkurrenten Dysenterie der Gallenausflub sistierte. 